\title{
ROL DEL INSTITUTO NACIONAL MATERNO PERINATAL EN LA REDUCCIÓN DE LA MORTALIDAD MATERNA Y NEONATAL
}

\author{
Enrique Guevara Ríos ${ }^{1,2, a}$
}

El 10 de octubre de 1826 el Mariscal don Andrés de Santa Cruz, quien, por decreto supremo emitido por Hipólito Unanue, creó la Casa de Maternidad, centro hospitalario que tendría como finalidad la atención de las parturientas, donde además se impartiría preparación en la práctica obstétrica para la formación de comadronas instruidas y hábiles ${ }^{1}$.

En 1943, durante el primer gobierno de don Manuel Prado, por decreto supremo, se le da la denominación oficial de Hospital de Maternidad de Lima. En 2002 logra la denominación de Instituto Especializado Materno Perinatal y desde el 2006 es categorizado como Instituto Nacional Materno Perinatal ${ }^{2}$.

La misión del INMP es brindar atención especializada a las mujeres, gestantes y recién nacidos de alto riesgo, desarrollando investigación, tecnologías y docencia a nivel nacional ${ }^{3}$. En su rol de establecimiento de tercer nivel tiene como políticas ${ }^{4}$ : el acceso oportuno a los servicios altamente especializados en las mujeres en la atención de la salud sexual y reproductiva y acceso a las gestantes y recién nacidos en el área materno perinatal, contribuyendo a la formación de redes de servicios efectivas para la atención, con énfasis en la atención de alta complejidad; el fortalecimiento de la capacidad resolutiva para la alta complejidad de los principales hospitales del país con atención materno perinatal, mejorando las competencias del talento humano; y promover la docencia e investigación materna perinatal, el desarrollo de nuevas tecnologías, innovación y modelos de atención.

EI INMP tiene como objetivo general Brindar atención altamente especializada y de calidad en salud sexual y reproductiva con énfasis en las gestantes y neonatos de alto riesgo que acudan al INMP y que sean referidas de otros establecimientos de salud de Lima y de todas las regiones del país. Sus objetivos específicos son:

1. Atención altamente especializada en obstetricia crítica, medicina materno-fetal, medicina reproductiva y en neonatología crítica.

2. Desarrollo del potencial humano de la institución, a nivel nacional e internacional.

3. Promoción de investigaciones que puedan contribuir a mejorar la salud de las mujeres, gestantes y neonatos en el país.

Para lograr el objetivo 1 se ha estandarizado de los procesos de atención mediante guías de atención basadas en evidencia científica en ginecología, obstetricia y neonatología; como la Guía de Práctica Clínica para la prevención y manejo de la preeclampsia y eclampsia, y la Guía de Práctica Clínica de Sepsis Neonatal, ambas elaboradas con el Método Agree II y Grade ${ }^{5}$. Se ha mejorado la capacidad resolutiva para la atención de casos de obstetricia y neonatología crítica mediante la implementación de la sala de shock trauma adulto/ neonato en el servicio de Emergencia, la actualización de las claves roja, azul y amarilla en un trabajo conjunto con OPS, el fortalecimiento de la UCI Materna I, la implementación de la UCIM II; y debido a la pandemia con COVID se ha implementado a la UCI Materna I para la atención de gestantes con COVID y compromiso

\footnotetext{
Instituto Nacional Materno Perinatal. Lima, Perú.

2 Departamento de Ginecología y Obstetricia. Universidad Nacional Mayor de San Marcos. Lima,Perú.

a Médico Ginecólogo-Obstetra. Director del Instituto Nacional Materno Perinatal. Coordinador de Asistencia Técnica del Instituto de Salud Popular. Consultor de Pathfinder International. Profesor Ordinario de la Facultad de Medicina, Universidad Nacional Mayor de San Marcos Lima-Perú.

Citar como: Guevara R. Rol del Instituto Nacional Materno Perinatal en la reducción de la mortalidad materna y neonatal. Rev Peru Investig Matern Perinat 2020; 9(4): 6-7

DOI https://doi.org/10.33421/inmp.2020219
} 
respiratorio severo. Se ha fortalecido la UCI Neonatal, el Banco de Leche Humana y se ha acreditado en el 2019 como un Hospital Amigo de los Niños/as ${ }^{6}$. El INMP está desde el 2015 en el proceso para acreditar como Hospital III-2 7; viene realizando rondas de seguridad en todos los servicios 8 ; se promueve la información de eventos adversos durante la atención y se realiza una supervisión diaria del desempeño de los equipos de guardia.

Para el objetivo 2 se realizan capacitaciones organizadas para la mejora de la calidad de la atención como han sido el Curso de Gestión Hospitalaria, el Curso Taller Formación de Evaluadores Internos, y el Curso Taller de Excelencia en Trato al Usuario; las capacitaciones organizadas para la implementación del uso de la Historia Clínica Perinatal SIP de "Mujeres en Situación de Aborto", utilizada por el CLAP; capacitación en la metodología para la implementación del Modelo de Atención Prenatal basado en el concepto de Pirámide Invertida; capacitación en reanimación cardiorespiratoria del adulto y neonato básica y avanzada; y capacitaciones en manejo de emergencias obstétricas y neonatales. Se han realizado asistencias técnicas directas en salud materno perinatal a diferentes regiones del país: Lima Provincias (Matucana, Jicamarca, Ricardo Palma), Ica (Hospital Santa María del Socorro de Ica, Hospital Regional de Ica, DIRESA Ica), La Libertad (Hospital Regional de Trujillo, Hospital Belén de Trujillo, Hospital de Apoyo de Huamachuco, Hospital de apoyo de Primavera), Hospital Regional de Ayacucho, Hospital Regional de Loreto, entre otros. Se han firmado 7 convenios con universidades para brindar apoyo a la docencia en pre y post grado. Se ha fortalecido la telemedicina a través de la cual entre el 2015 - 2019 han sido capacitados cerca de 10,000 profesionales de la salud. Durante la pandemia por COVID y desde Mayo del 2020 se han realizado más de 6,000 consultas por telemedicina especialmente de atención prenatal ${ }^{9}$.

En su rol de instituto ha organizado y desarrollado el 3er Congreso Internacional Salud Materno Perinatal "Avances y nuevas evidencias" en el $2015^{10}$; el 4to Congreso Internacional Salud Materno Perinatal "Innovaciones y seguridad en la atención” en el $2017^{11}$; y el 5to Congreso Internacional Salud Materno Perinatal "Rumbo al bicentenario" en el $2019{ }^{12}$. Todos ellos con la finalidad de actualizar los conocimientos y compartir las experiencias del instituto para mejorar las competencias del personal de salud a nivel nacional.

Finalmente, en el objetivo 3 , se ha fortalecido la Unidad de Investigación con una mejora de la infraestructura y mejora en los recursos humanos dedicados exclusivamente a la investigación. Asimismo se ha logrado que la Revista Peruana de Investigación Materno Perinatal (Rev Peru Investig Matern Perinat, ISSN 2663113X), que es el órgano oficial de difusión científica del Instituto Nacional Materno Perinatal (INMP), se encuentra Indexada en Latindex desde diciembre del 2015 y en la actualidad se está postulando para SciELO. La revista tiene como objetivo difundir la producción científica de la especialidad materno perinatal/neonatal y de salud sexual y reproductiva entre sus integrantes, profesionales interesados y de especialidades afines, con la finalidad de contribuir a mejorar la situación de salud materno perinatal del país y de la región ${ }^{13}$.

En conclusión, el INMP es una institución de alta complejidad y su rol es de contribuir a la mejora de la salud materna, mediante la disminución de los casos de mortalidad materna y neonatal y la recuperación de los casos de morbilidad materna extrema. Esto se logra a través de una adecuada gestión de la capacidad resolutiva del establecimiento, así como potenciar el recurso humano, cerrando la brecha y mejorando sus competencias. Finalmente, la investigación materno perinatal es fundamental para dirigir los escasos recursos a la priorización de las principales complicaciones del embarazo, parto, y puerperio y de los recién nacidos. Todo esto es posible solo con una adecuada ejecución presupuestal, transparente, sin corrupción y pensando en la salud de todas/os los peruanos. La salud de las gestantes y los recién nacidos debe ser una prioridad nacional ya que es un derecho humano básico y fundamental.

Financiamiento: Autofinanciado.

Conflicto de interés: El autor declara no tener algún conflicto de interés.

\section{REFERENCIAS BIBLIOGRÁFICAS}

1. Huamán J, Bazul V, Miranda C. Maternidad De Lima: Un Hospital "Nómade" Cuna De La Obstetricia Peruana". Ginecología y Obstetricia - Vol. 39 №15 Setiembre 1993. https://sisbib.unmsm.edu.pe/bvrevistas/ ginecologia/ vol 39n15/maternidad lima.htm

2. Instituto Nacional Materno Perinatal Oficina de Epidemiología y Salud Ambiental. Análisis de la situación de los servicios hospitalarios del Instituto Nacional Materno Perinatal (INMP) - Maternidad de Lima. 2012.

3. https://www.inmp.gob.pe/institucional/mision-yvision/1415392243

4. https://www.inmp.gob.pe/institucional/ presentacion/1415392103

5. https://www.inmp.gob.pe/ busqueda?url=portal\&busqueda=guias

6. https://www.inmp.gob.pe/ noticias? texto $=$ obstetricia $\&$ dia $=31 \&$ mes $=10 \&$ anio $=2020$

7. https://www.inmp.gob.pe/ busqueda?url=portal\&busqueda=acreditaci $\% \mathrm{C} 3 \% \mathrm{~B} 3 \mathrm{n}$

8. https://www.inmp.gob.pe/ busqueda?url=portal\&busqueda=rondas

9. https://www.inmp.gob.pe/institucional/red-nacional-detelesalud-materno-perinatal/1488812216

10. https://www.inmp.gob.pe/institucional/3er-congresointernacional-salud-materno-perinatal-avances-y-nuevasevidencias/1440792541

11. https://www.inmp.gob.pe/institucional/programa/1506629839

12. https://www.inmp.gob.pe/institucional/ presentacion/1566841248

13. https://www.inmp.gob.pe/institucional/numerosdisponibles/1422469844 